UCRL-JC-130683

PREPRINT

\title{
Cluster Ion Beam Polishing for Inertial Confinement Fusion Target Capsules
}

\author{
R. McEachern, J. Matsuo, I. Yamada
}

This paper was prepared for submittal to the 12th International Conference on Ion Implantation Technology Kyoto, Japan

June 22-26, 1998

June 9,1998

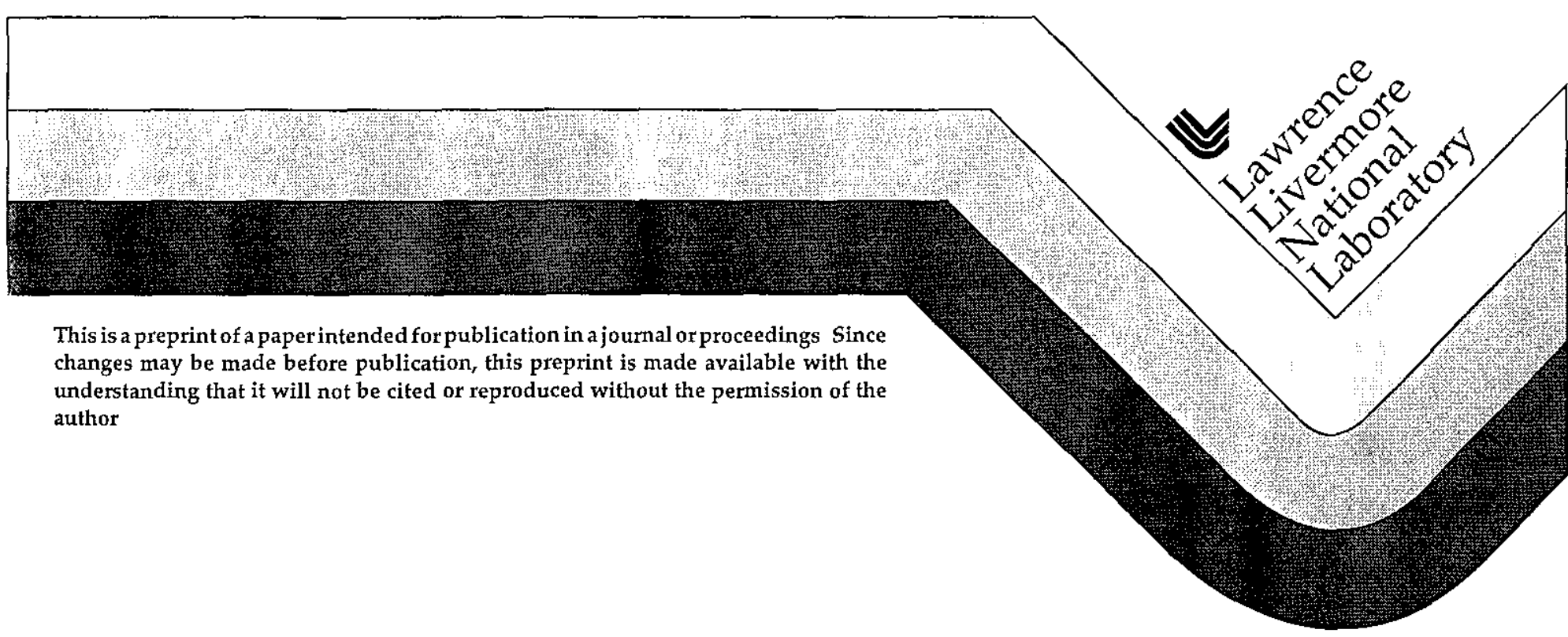




\section{DISCLAIMER}

This document was prepared as an account of work sponsored by an agency of the United States Government. Neither the United States Government nor the University of California nor any of their employees, makes any warranty, express or implied, or assumes any legal liability or responsibility for the accuracy, completeness, or usefulness of any information, apparatus, product, or process

disclosed, or represents that its use would not infringe privately owned rights. Reference herein to any specific commercial product, process, or service by trade name, trademark, manufacturer, or otherwise, does not necessarily constitute or imply its endorsement, recommendation, or favoring by the United States Government or the University of California. The views and opinions of authors expressed herein do not necessarily state or reflect those of the United States Government or the University of California, and shall not be used for advertising or product endorsement purposes. 


\title{
Cluster Ion Beam Polishing for Inertial Confinement Fusion Target Capsules
}

\author{
R. McEachern 1, J Matsuo 2 and I. Yamada2 \\ 1) Lawrence Livermore National Laboratory \\ Livermore, CA 94550 USA \\ rlm@linl gov \\ 2) Ion Beam Engineering Experimental Laboratory, Kyoto University \\ Sakyo, Kyoto 606, Japan \\ i-yamada@kuee kyoto-u ac jp
}

\begin{abstract}
Targets for Inertial Confinement Fusion (ICF) typically consist of a hollow, spherical capsule filled with a mixture of hydrogen isotopes. Typically, these capsules are irradiated by short, intense pulses of either laser light ("direct drive") or laser-generated $x$-rays ("indirect drive"), causing them to implode This compresses and heats the fuel, leading to thermonuclear fusion. This process is highly sensitive to hydrodynamic (e g, Rayleigh-Taylor) instabilities, which can be initiated by imperfections in the target. Thus, target capsules must be extremely spherical and smooth One of the lead capsule designs for the National Ignition Facility, a $18 \mathrm{MJ}$ laser being built at Livermore, calls for a $2-\mathrm{mm}$ diam capsule with a $150-\mu \mathrm{m}$-thick copper-doped beryllium wall. These capsules can be fabricated by sputter depositing the metal onto a spherical plastic mandrel. This results in surfaces with measured Rq's of 50 to 150 $\mathrm{nm}$, as measured with an atomic force microscope For optimal performance the roughness should be below 10 $\mathrm{nm} \mathrm{rms}$ We have begun studying the use of ion cluster beam polishing as a means of improving the surface finish of as-deposited capsules In this approach, a batch of capsules would be agitated in a bounce pan inside a vacuum chamber during exposure to the cluster beam. This would ensure a uniform beam dose around the capsule. We have performed preliminary experiments on both $\mathrm{Be}$ flats and on a stationary Be capsule On the capsule, the measured $\mathrm{Rq}$ went from $64 \mathrm{~nm}$ before polishing to 15 $\mathrm{nm}$ after This result was obtained without any effort at process optimization. Similar smoothing was observed on the planar samples
\end{abstract}

This work was performed under the auspices of the $U$. S. Department of Energy by Lawrence Livermore National Laboratory under contract No. W-7405-Eng-48. 
Introduction

Hydrodynamic instabilities during implosions of inertiallyconfined fusion (ICF) capsules are expected to play a dominant role in determining overall capsule performance. The ablation front is Rayleigh-Taylor (RT) unstable due to hot, low-density plasma pushing against and accelerating the cooler, denser shell. During this acceleration, surface imperfections grow at the ablation front and can, if large enough, lead to an asymmetric implosion or even shell break-up. Further RT growth degrades the final symmetry of the implosion and creates mix at the fuel-capsule interface, potentially decreasing burn efficiency or preventing ignition. Figure 1 shows a simplified schematic of this process. The National Ignition Facility (NIF), a 192-beam, 1.8 MJ laser facility under construction at Lawrence Livermore National Laboratory will require 2-mm-diam target capsules with stringent requirements on the surface finish. Surface roughness as low as 10 $\mathrm{nm}$ rms may be required.

A leading capsule design requires a 120 to $150-\mu$ m-thick $\mathrm{Cu}$ doped Be shell. One method being studied for fabricating these shells entails sputter deposition of Be onto a spherical mandrel. As deposited, such coatings are fairly rough-patch measurements with an atomic force microscope (AFM) typically yield $R_{q}$ measurements in the vicinity of $100 \mathrm{~nm}$. Work is continuing to improve the surface finish via process modifications, but it is clear that some method of polishing the finished capsule would be highly desirable.

A new polishing technique has been developed that is particularly promising for this application. It involves the use of ionized, accelerated clusters of noble gas atoms to bombard a surface. Typical cluster sizes are in the range of hundreds to a few thousand atoms, with ion energies of tens of $\mathrm{keV}$. Because of the 
still evident in Fig. 5b, even though the larger-scale topography has been greatly reduced. Figure 6 shows before and after AFM images taken from the pole of the spherical sample. The initial roughness was much greater, $64 \mathrm{~nm} \mathrm{rms}$, but significant improvement was still achieved (down to $14 \mathrm{~nm} \mathrm{rms).} \mathrm{This} \mathrm{sample}$ also demonstrates the importance of the angle of incidence of the clusters. Figure 7a shows an SEM image of the capsule surface prior to cluster ion bombardment. Figure $7 \mathrm{~b}$ shows a region near the pole (as in Fig. 6b), while Fig. 7c shows an image taken roughly $45^{\circ}$ down the side of the capsule. There is clearly an increase in the roughness relative to the pole, a phenomenon which has been observed in angle-of-incidence studies.

\section{Conclusions}

Cluster ion beam polishing is a promising technology for improving the surface finish of beryllium target capsules for ICF applications. As compared to conventional polishing, the technique offers the benefits of compatibility with the fabrication process (i.e., cluster bombardment could be applied to batches of capsules agitated in a bounce pan) and freedom from contamination.

Although significant smoothing of sputter-deposited Be films has been observed, polishing of capsules represents a unique challenge, since smoothing and roughening occur simultaneously. The portion of the capsule where the clusters are incident normal to the surface becomes smoother, while those areas subject to glancing bombardment become rougher. It is likely that the reduced sputter yield at such glancing angles will result in net smoothing when this technique is applied to a moving capsule, but this remains to be tested. 
high mass and energy but low velocity of these clusters, they are particularly efficient at removing atoms from the near-surface region without the deep implantation characteristic of monomer ions in this energy range. Sputtering yields of up to two orders of magnitude higher than for monomer ions have been observed, as well as lateral sputtering phenomena (see Fig. 2). The latter process plays a significant role in preferentially removing surface asperities.

Experiment

To evaluate cluster polishing on materials of interest to the ICF program, we used both planar and spherical Be samples. The planar sample was a $\sim 5-\mu \mathrm{m}$-thick layer of Be sputter-deposited onto an as-received Si wafer. The spherical sample was a 1-mmdiam plastic shell with an $80-\mu \mathrm{m}$-thick coating of Be. The spherical sample was glued to an aluminum SEM stub, so only the pole was bombarded normal to the surface.

The clusters are produced by expanding Ar through a Laval nozzle into a high-vacuum chamber following collimation by a skimmer. Ions are formed by electron-impact ionization, and an electrostatic retarding potential is used to mass-select the clusters. Figure 3 shows a schematic view of the apparatus. Both samples were subjected to a dose of $5 \times 1016$ ions $/ \mathrm{cm}^{2}$ of argon cluster ions at $20 \mathrm{keV}$. The samples were measured before and after exposure with a Digital Instruments Dimension 3000 AFM.

\section{Results}

Figure 4 shows $5-\mu \mathrm{m}$-square AFM images of the planar sample before and after cluster ion bombardment. Prior to exposure, the roughness of the surface was $18 \mathrm{~nm} \mathrm{rms,} \mathrm{and} \mathrm{after,}$ it improved to $3.6 \mathrm{~nm}$. Figure 5 shows the same surface, but at $5 \mathrm{X}$ higher magnification. Note that the substructure visible in Fig. $5 \mathrm{a}$ is 


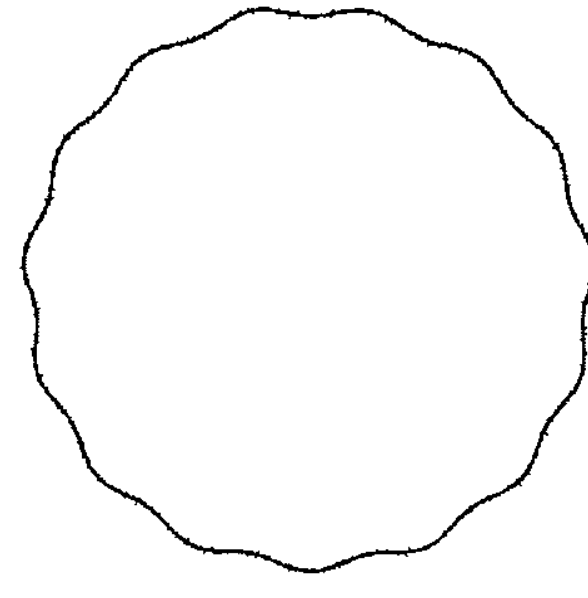

initial surface (with some random roughness)
Outer surface of capsule is RayleighTaylor unstable during the implosion

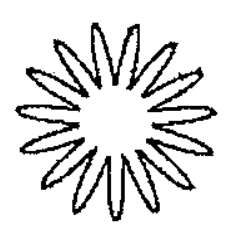

final surface at maximum compression

Fig. 1. A cartoon showing the growth of surface imperfections during the implosion of an ICF capsule due to hydrodynamic instabilities. 
Low charge-to-mass ratio

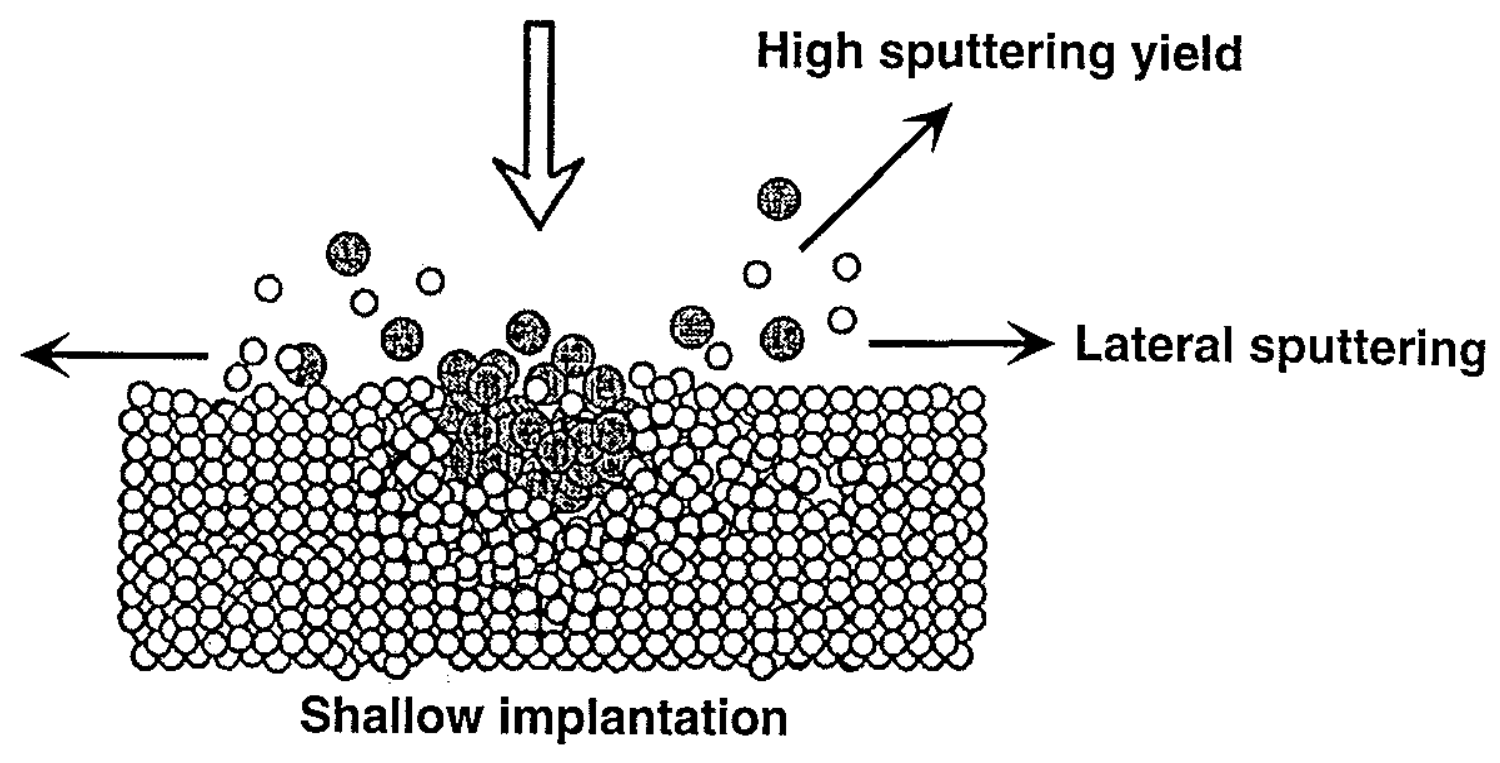

Fig. 2. Typical cluster-surface interactions important for polishing applications.

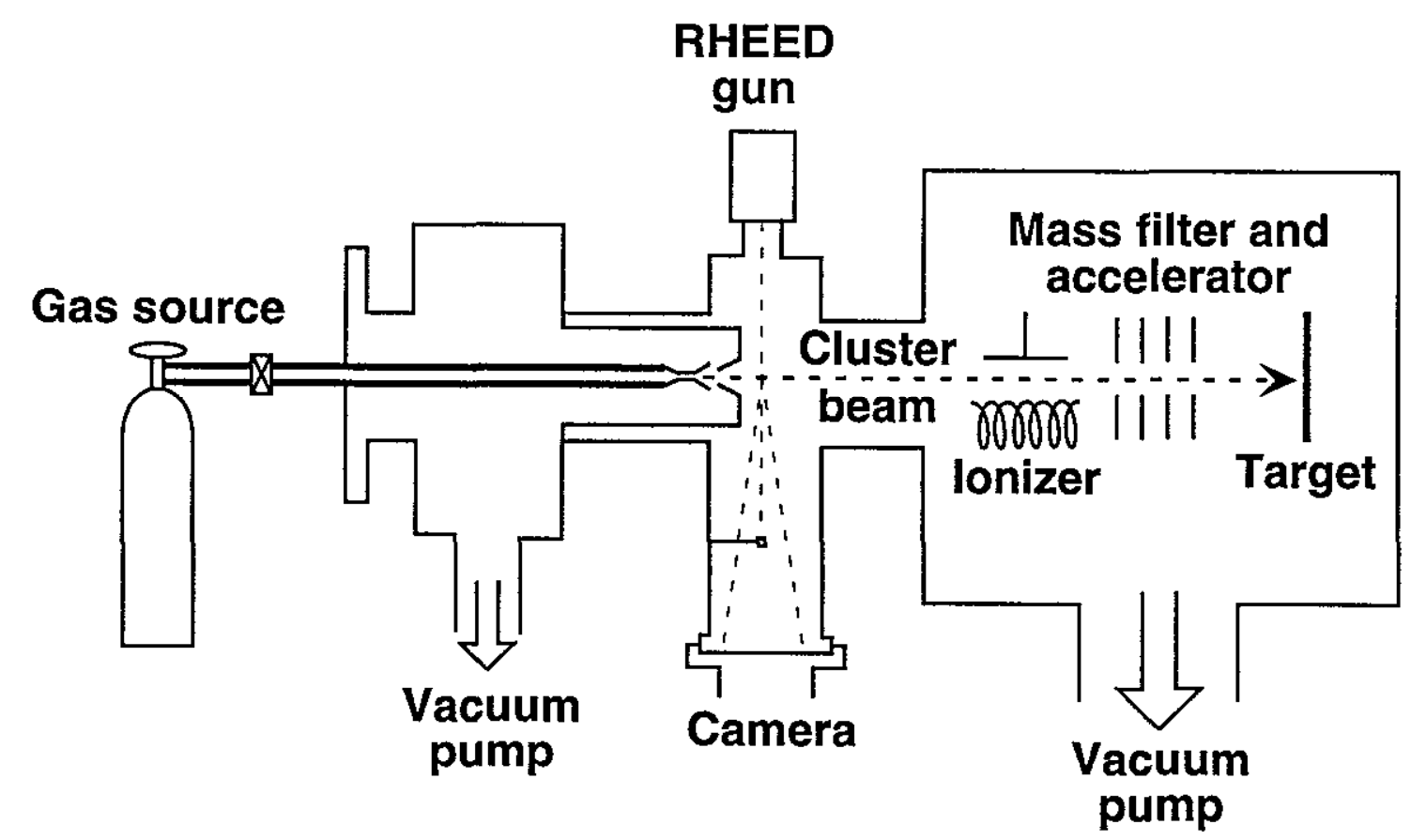

Fig. 3. A schematic diagram of the cluster ion beam source. Argon was the source gas in the present experiment. 

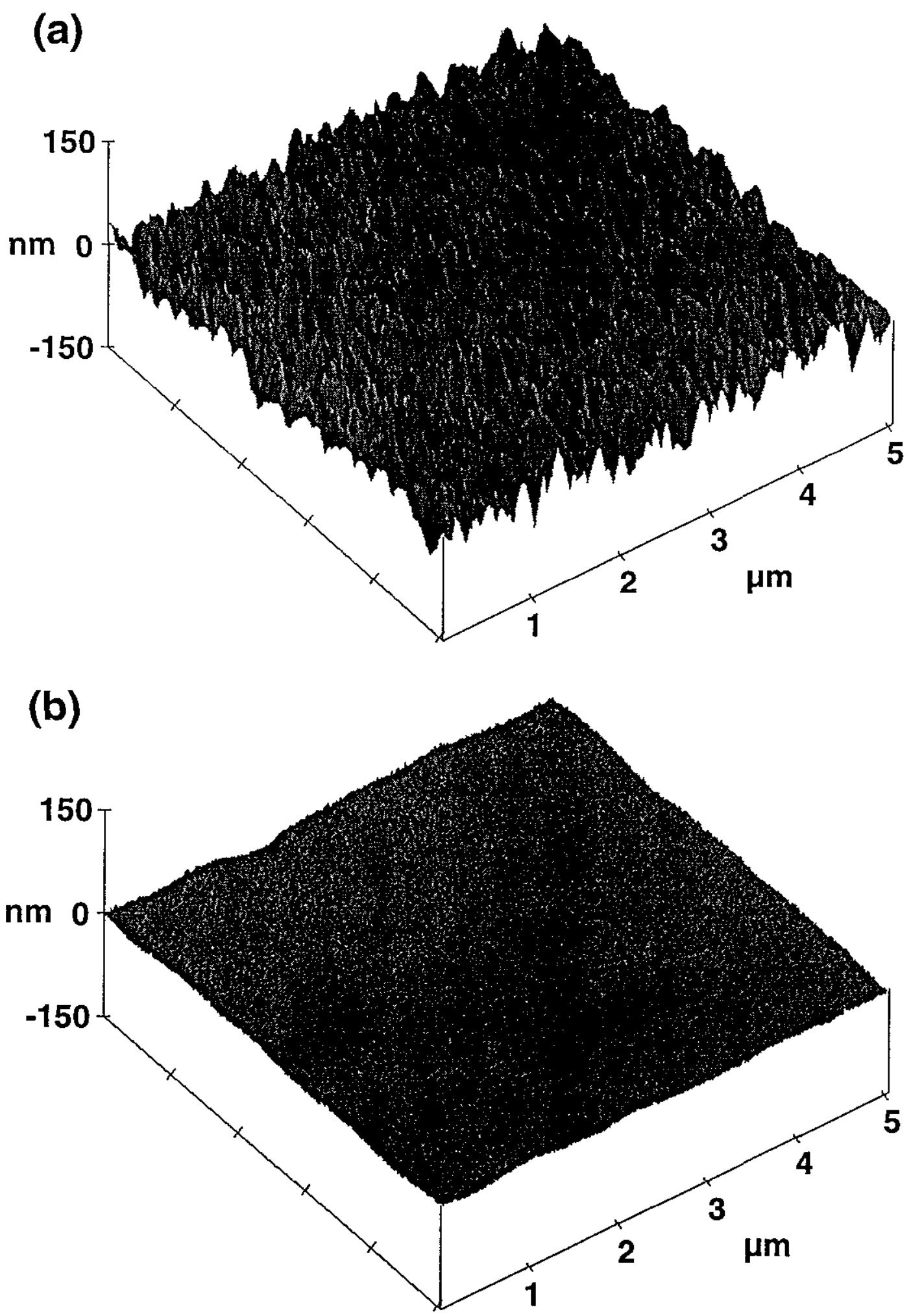

Fig. 4. AFM images of a Be film sputter-deposited onto a silicon substrate. a) as-deposited; $R_{\mathrm{q}}=18 \mathrm{~nm}$ b) after exposure, to $5 \times 10^{16}$ (ONS/ a Ar cluster ions at $20 \mathrm{keV} ; \mathrm{R}_{\mathrm{q}}=3.6 \mathrm{~nm}$ 
(a)

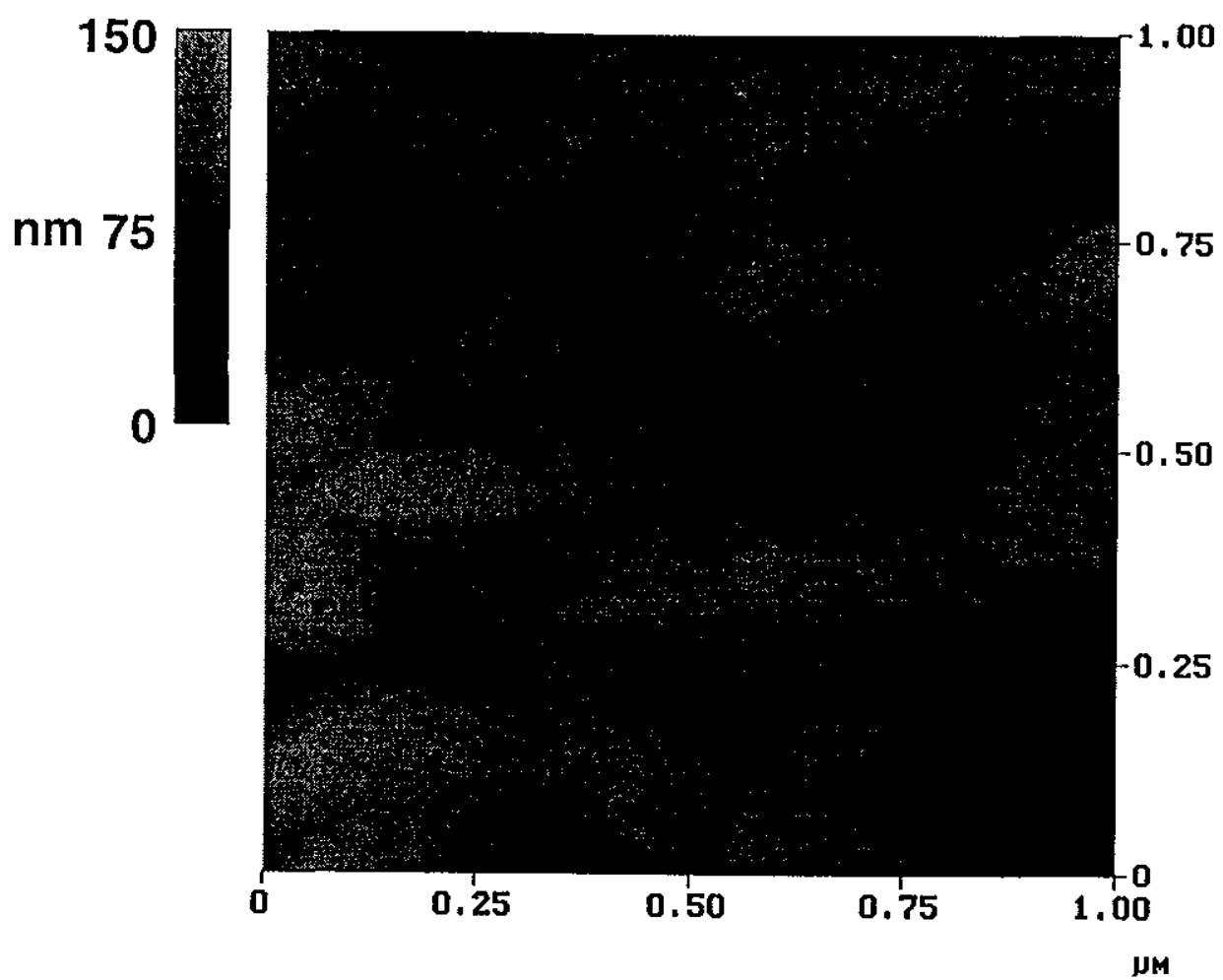

(b)

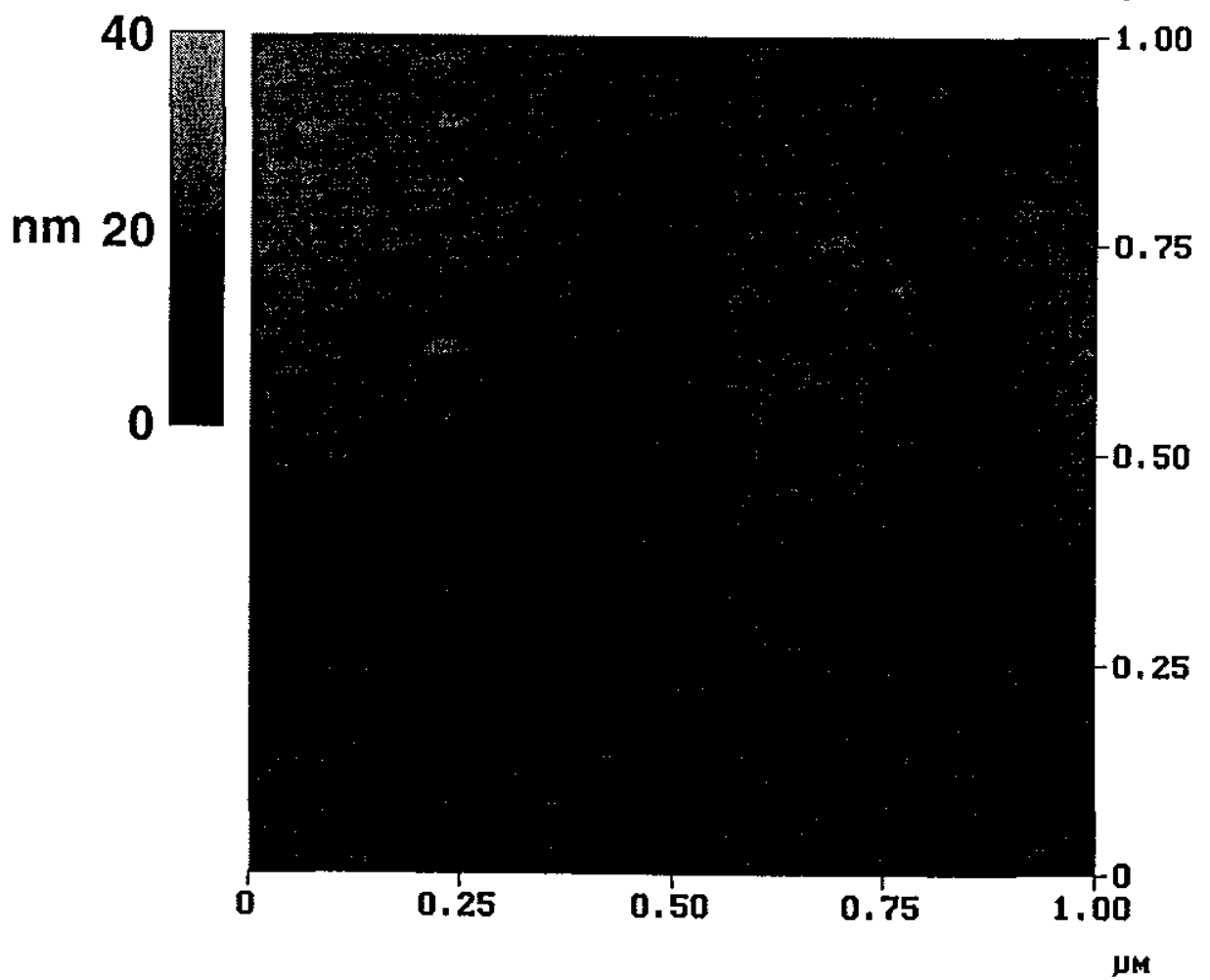

Fig. 5. Higher-magnification images of the film in Fig. 4. Note that the color scale is different for the two images. a) as-deposited b) after exposure to $5 \times 10^{16} \mathrm{Ar}$ cluster ions at $20 \mathrm{keV}$ 

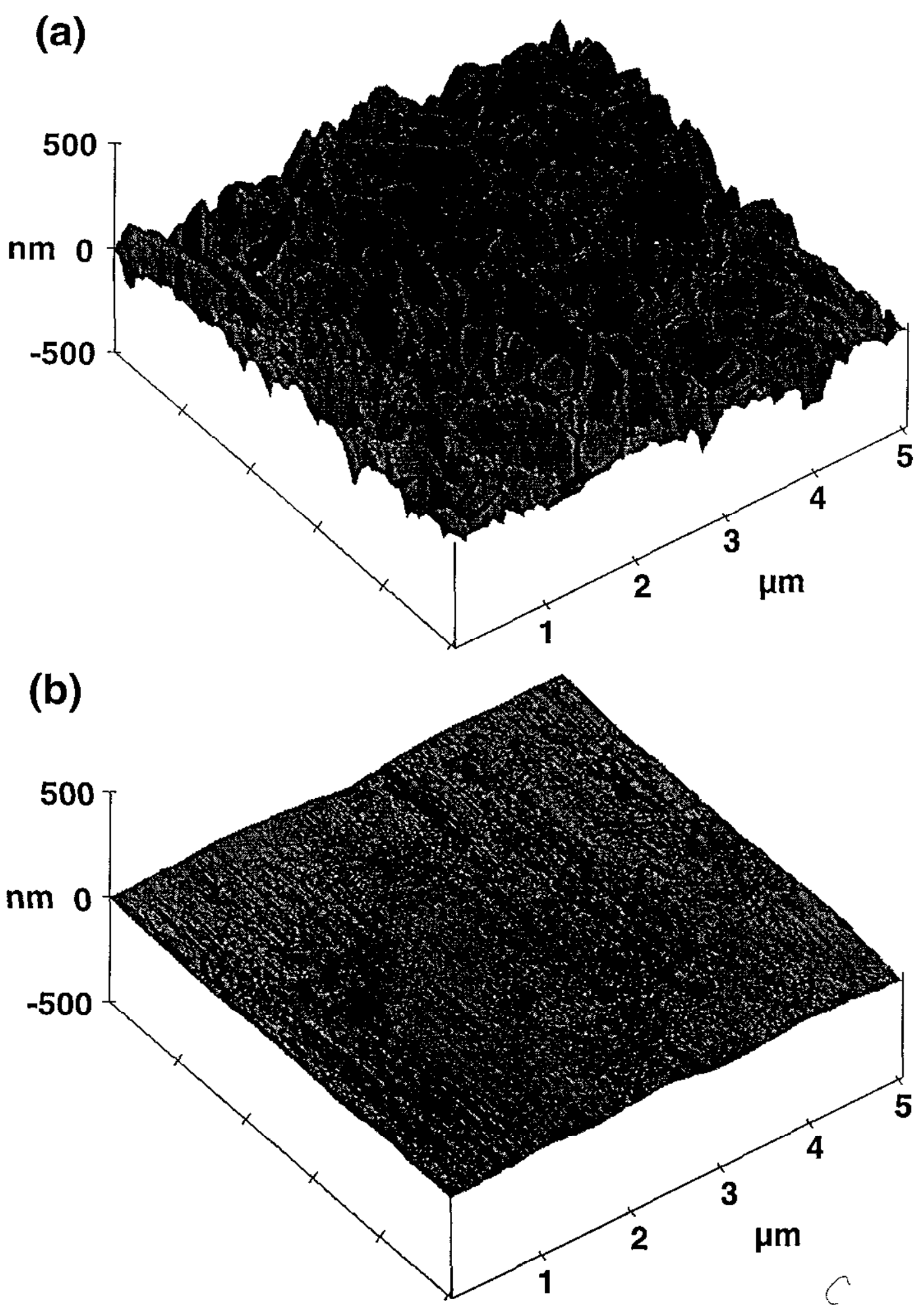

Fig. 6. AFM images of a Be film sputter-deposited onto 1-mm-diam plastic capsule. The images were taken at the pole of the capsule, where the cluster ions were incident normal to the surface.

a) as-deposited; $R_{\mathrm{q}}=64 \mathrm{~nm} \mathrm{~b}$ ) after exposure to $5 \times 10^{16}$ Ar cluster ions at $20 \mathrm{keV} ; \mathrm{R}_{\mathrm{q}}=14 \mathrm{~nm}$ 


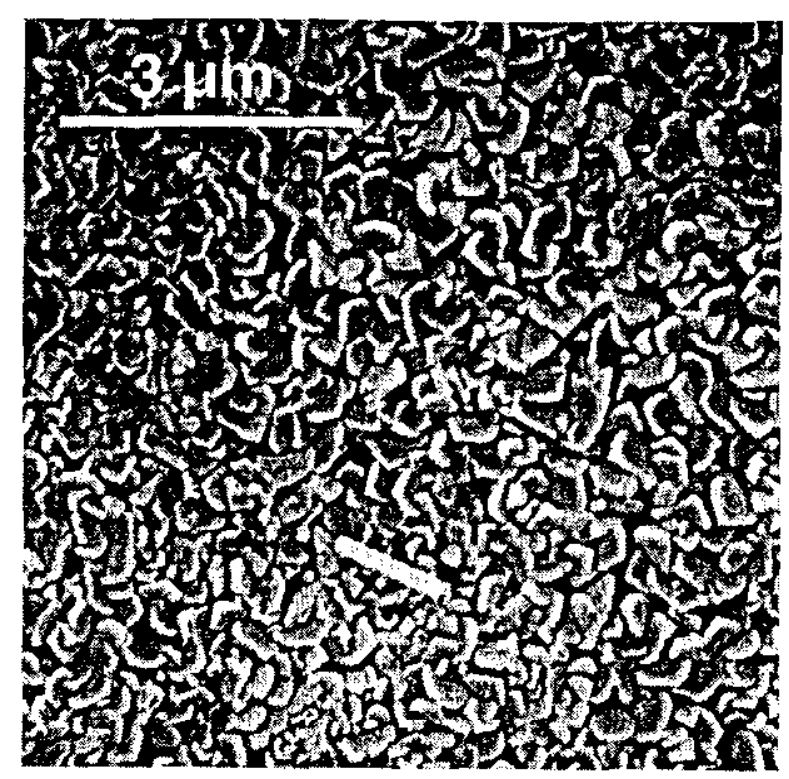

(a) Prior to cluster ion bombardment

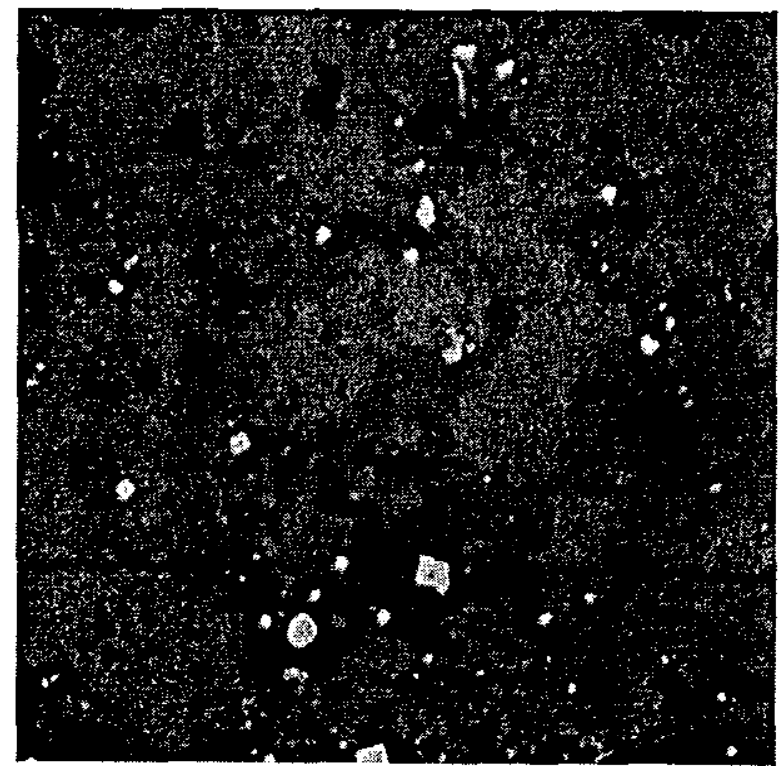

(b) The pole of the capsule after exposure to cluster ions

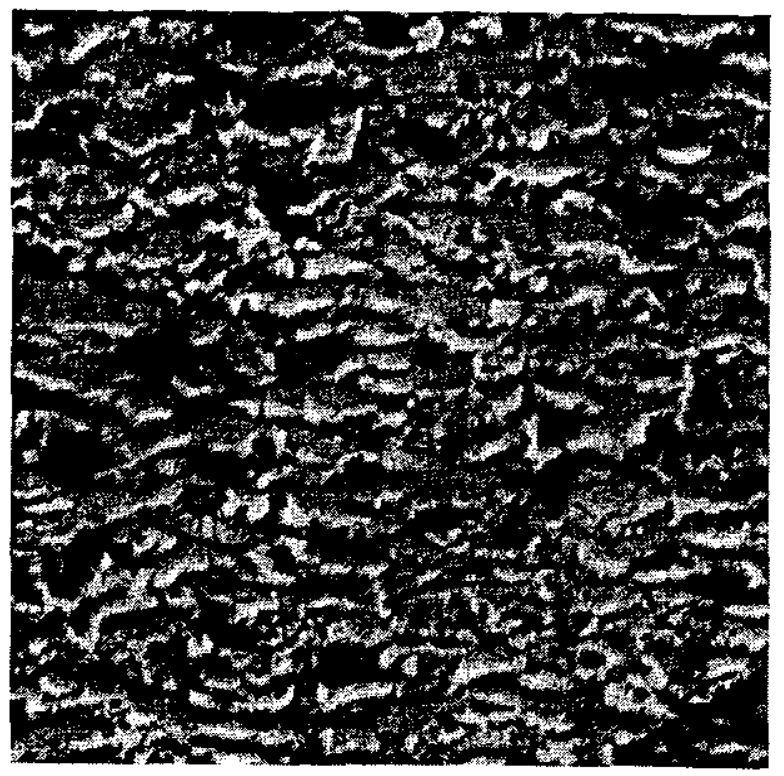

(c) Approximately $45^{\circ}$ off the pole after exposure to cluster ions

Fig. 7. SEM images of the Be-coated capsule from Fig. 6. 


\section{What is Inertial Confinement Fusion (ICF)?}

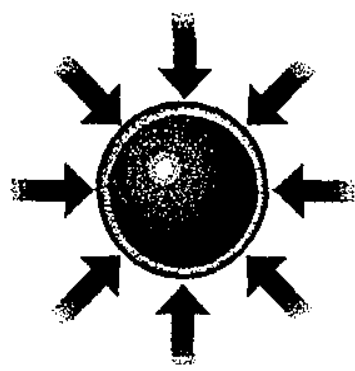

Atmosphere formation

$X$ rays rapidly heat the surface of the fusion capsule forming a surrounding plasma envelope.

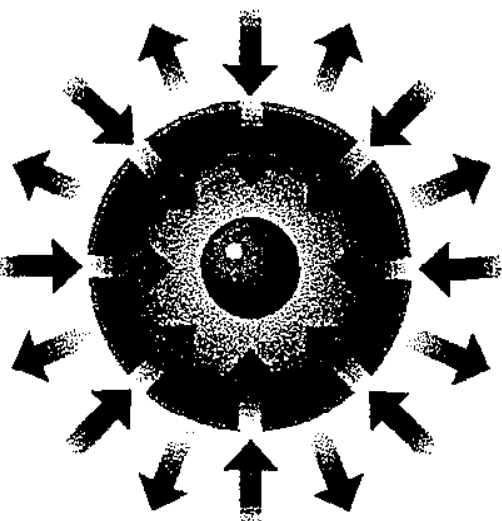

Compression

Fuel (deuteriumtritium) is compressed by the rocket-like blowoff of the hot surface material.

$\rightarrow$ Motion of capsule wall

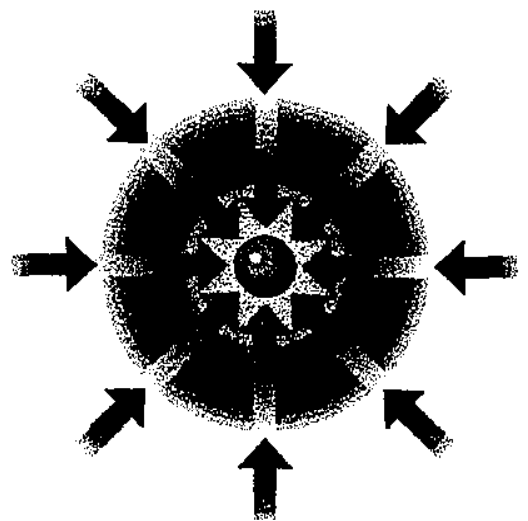

Ignition

During the final part of the laser pulse, the fuel core reaches 20 times the density of lead and ignites at $100,000,000^{\circ} \mathrm{C}$.

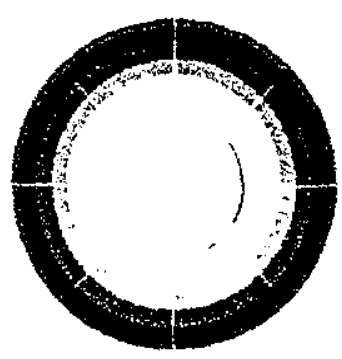

Burn

Thermonuclear burn spreads rapidly through the compressed fuel, yielding many times the input energy. 


\section{How is indirect drive ICF achieved?}
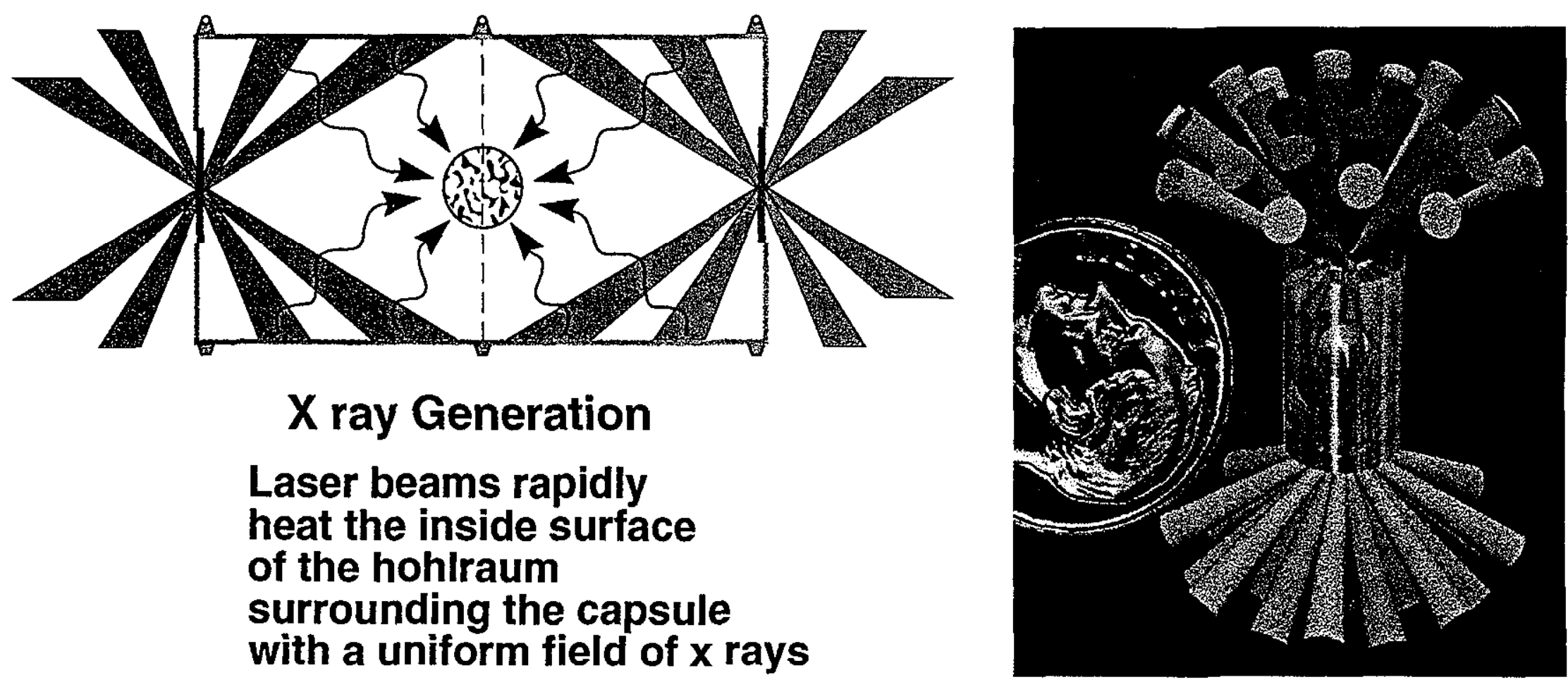

Laser beams rapidly heat the inside surface of the hohiraum surrounding the capsule with a uniform field of $x$ rays 


\section{What will an ICF capsule for the National Ignition Facility look like?}

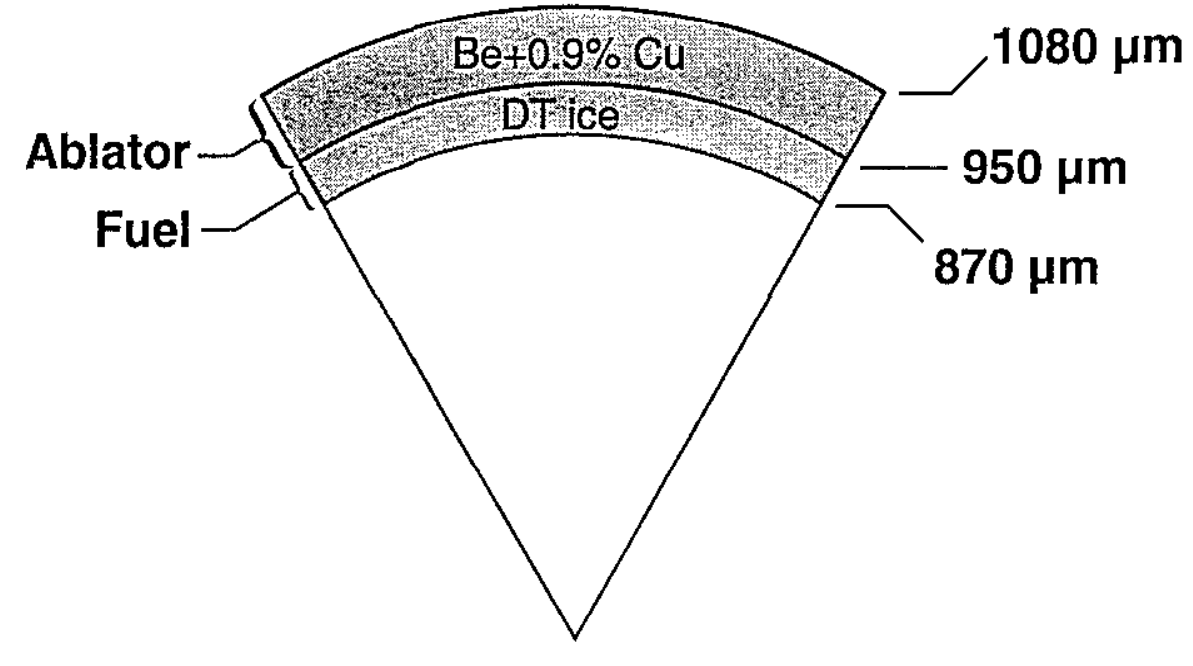

Sputter targets

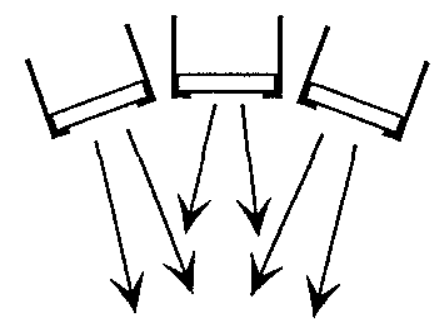

Bounce pan
One of the leading capsule designs requires an ablator composed of beryllium doped with 0.9 at. $\% \mathrm{Cu}$. There are other designs that use plasma polymer or polyimide ablators. The capsule shown here is designed to produce $17.1 \mathrm{MJ}$ of fusion yield under the following conditions:

Laser drive: 1.3 MJ

Hohlraum temperature: $300 \mathrm{eV}$

These capsules can be fabricated by sputter-depositing Be onto thin-walled plastic capsules, or mandrels. These mandrels are continuously agitated in a pan underneath the sputter sources, ensuring a uniform coating. 


\section{The ICF process is hydrodynamically unstable}

The interface between a low-density fluid pushing against and accelerating a high-density fluid is prone to Rayleigh-Taylor instabilities

Example: A light fluid supporting a heavy fluid against gravity

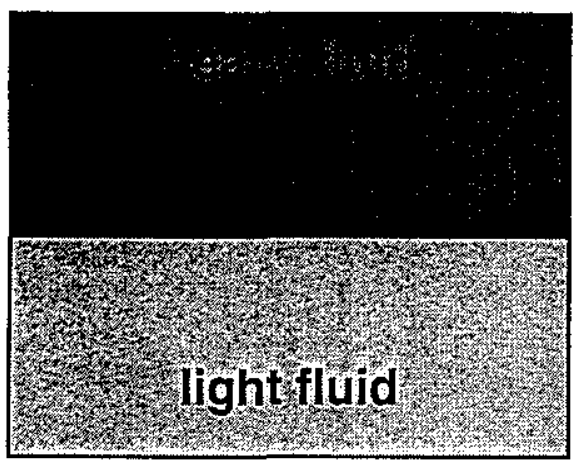

A flat interface between the liquids is metastable

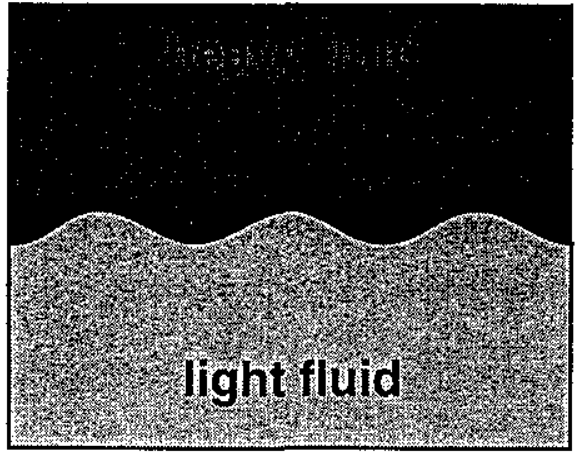

A small perturbation develops...

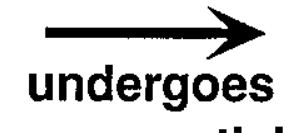

exponential growth...

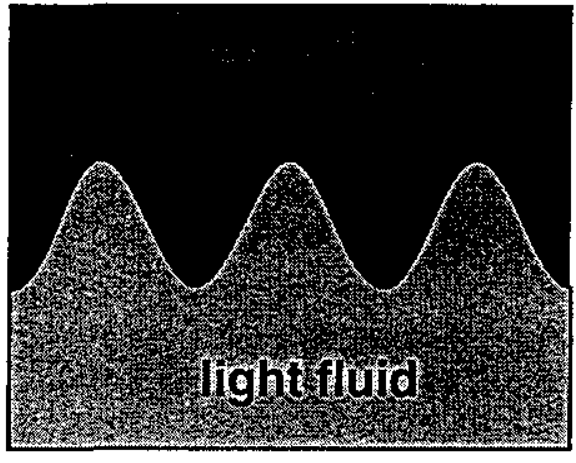

and becomes a large perturbation

During an ICF implosion hot, low density material ablates and pushes against the heavier, cooler shell 


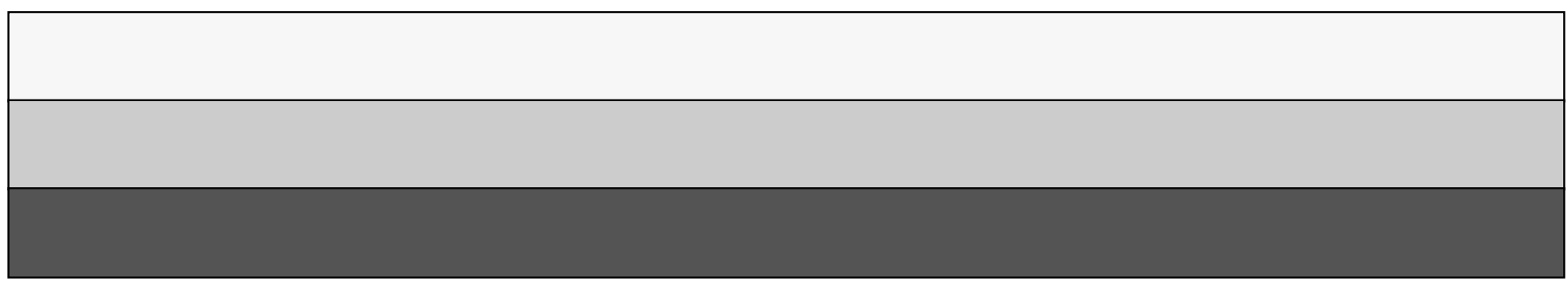

